Research Article

\title{
Optimal Design Parameters of a Percussive Drilling System for Efficiency Improvement
}

\author{
Changheon Song, ${ }^{1,2}$ Jintai Chung, ${ }^{2}$ Jae-Sang Cho, ${ }^{3}$ and Yun-Joo Nam $\mathbb{D}^{1}$ \\ ${ }^{1}$ Korea Institute of Industrial Technology, Gyeongsan-si, Republic of Korea \\ ${ }^{2}$ Department of Mechanical Engineering, Hanyang University, Ansan-si, Republic of Korea \\ ${ }^{3}$ R๘D Center, Soosan Heavy Industries Co., Ltd., Hwaseong-si, Republic of Korea \\ Correspondence should be addressed to Yun-Joo Nam; yjnam@kitech.re.kr
}

Received 16 June 2017; Revised 9 August 2017; Accepted 10 October 2017; Published 5 February 2018

Academic Editor: Fernando Lusquiños

Copyright (c) 2018 Changheon Song et al. This is an open access article distributed under the Creative Commons Attribution License, which permits unrestricted use, distribution, and reproduction in any medium, provided the original work is properly cited.

\begin{abstract}
This paper aims to determine the optimal design parameters for percussive drilling systems considering the bit-rock interaction. First, the motion dynamics of a bit impacted by a dropped piston are modeled by impact stress propagation and a rock-breaking mechanism representing the penetration resistance coefficient and unloading constant. Next, the damping vibration behavior of the bit is investigated considering the impact duration and the rock loading/unloading condition. In addition, the proposed dynamics are simplified by adopting two dimensionless parameters representing the bit-piston mass ratio and the rock-piston stiffness ratio. Finally, the drilling efficiency, defined by the energy transmitted from the piston to the rock, is presented in terms of the proposed parameters. The use of optimal design parameters for percussive drilling systems improved the drilling efficiency. These results are applicable to the design and performance estimation of down-the-hole and top-hammer systems.
\end{abstract}

\section{Introduction}

Drilling equipment is the collective term used for machines that apply impact and rotation forces to drill (for the most part) surfaces and blastholes, and it is classified as tophammer drilling (THD), down-the-hole (DTH) drilling, and rotary drilling $(\mathrm{RD})$ rigs, depending on the operating method (Figure 1). In general, THD is used mostly for mining and civil blasting works, where the rig drills into the earth usually to a depth of $1-20 \mathrm{~m}$ and at most $40 \mathrm{~m}$; DTH is used mainly for groundwater development and can create holes to a maximum depth of $4000 \mathrm{~m}$; and $\mathrm{RD}$ bores the deepest holes, most commonly for petroleum gas extraction and geothermal development, being propelled by its own weight to reach depths of up to $10,000 \mathrm{~m}$ [1].

Simply put, a percussive drilling mechanism utilizes the percussive energy resulting from the repeated impact of the drifter (THD rigs) or the DTH hammer (DTH rigs) and the feed force and rotation force that are transmitted to the drill bit through the drill rod. The energy generated from the repeated impacts is then converted into wave energy, which is transmitted to the rock via the drill bit. Finally, the drill bit, now with enough impact energy for drilling, cuts into and crushes the rock. The rate at which the impact-generated energy in a percussive drilling system is transmitted (i.e., the drilling efficiency) is determined by complex effects such as drilling rod, coupling sleeve, the compressive strength of the rock, and interactions between the drill bit and the rock. Therefore, certain drilling methods are highly efficient, with high rates of penetration, when drilling soft rock (uniaxial compressive strength, UCS, $<20 \mathrm{MPa}$ ) or medium hard rock (UCS 50-120 MPa), but the efficiency decreases when drilling very hard rock (UCS > $200 \mathrm{MPa}$ ) [2].

There are numerous previous studies regarding drill bit, rock drilling, the transmission of impact energy, and drilling efficiency. Hustrulid and Fairhurst [3-6] investigated energy transmission between the drill steel and rock and measured the specific energy resulting from the impact force.

Chiang and Elias [7] used the finite element method (FEM) to study factors such as energy transmission, 


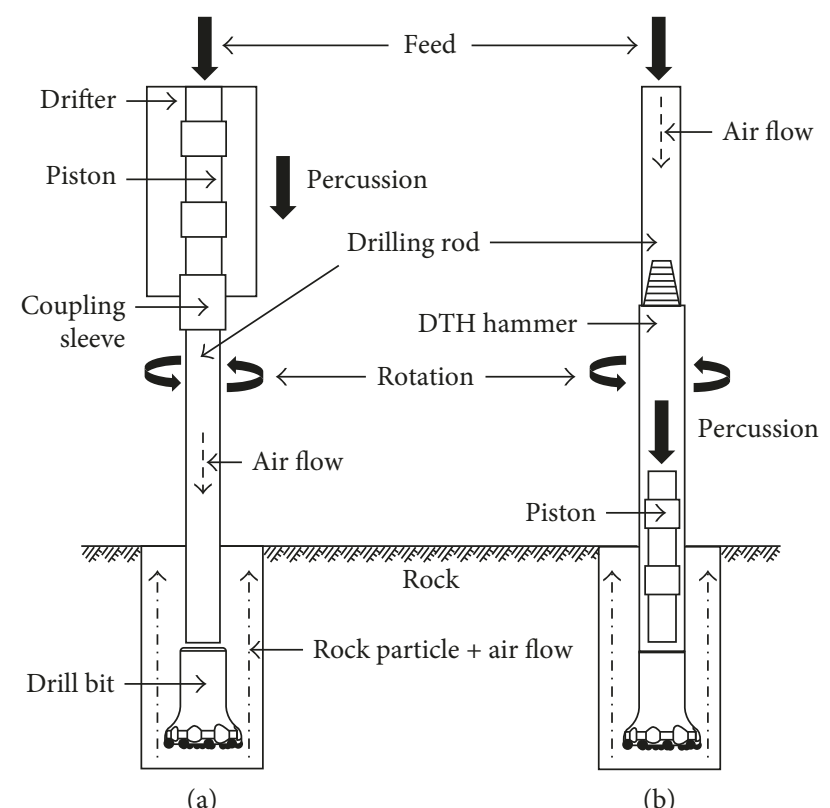

(a)

(b)

FIgURE 1: Drilling mechanisms of two types of percussive drilling system: (a) top-hammer drilling and (b) down-the-hole drilling (modified from Song et al. [1]).

interactions between the drill bit and rock, and the rock penetration process, which are important in the research and evaluation of drill equipment. Song et al. [1] proposed optimal design factors and range for top-hammer drill bit based on multiphase flow simulation and response surface methodology (RSM). Also, Song et al. [8] designed a labscale testing system to test drilling performance of a drill bit and investigated the effect of bit design factors on the performance of drilling. Kwon et al. [9] validated the effect of drill bit button arrangement on drilling efficiency through a piston percussion test using piston drop testing system. Lundberg and Okrouhlik [10] conducted a study of the 3D effects on the efficiency of three representative methods for percussive rock drilling (hammer drilling, DTH drilling, and churn drilling) in which the efficiency of various drilling methods was compared via 1D analysis and 3D FEM. Lundberg and Collet [11] investigated the optimal incident wave that maximizes the conversion efficiency of the wave energy in the THD method. All these prior studies essentially considered the transmission of impact energy from the piston and drill bit. However, they failed to examine the effect of the dynamic properties of the drill bit resulting from the piston impact. Li et al. [2] analyzed the piston reaction force characteristics of percussive hammers using the stress wave propagation theory and the law of conservation of energy and also studied the energy transmission efficiency of a DTH hammer tool [12]. The studies by Li et al. [2, 12] considered the dynamics of the drill bit resulting from the piston impact. However, this consideration only addressed the underdamped modes of the system response characteristics that result from the rotational movement of the drill bit and the effect of the rock. The work; therefore, neglected the dynamics of the drill bit generated under various other damped modes. The study was further limited in that it employed an excessively large range of rock-to-piston stiffness ratios $(\beta)$ in the transmission efficiency of impact energy. Overall, while many studies have investigated rock drilling and the transmission of impact energy, few have addressed the damping characteristics generated from the penetration process, the rock effect, and the movement of the drill parts or investigated the related drilling efficiency.

The analysis of behavior of drill bit should be performed for the design and manufacturing of a percussive drilling system with high drilling efficiency because the drilling efficiency and the range of rock fracturing depend on movement characteristics of drill bit during percussion and because drill bit also percusses the rock directly, meaning the close relationship with the delivery of percussion energy and drilling efficiency.

This study aimed to identify design parameters that could optimize the drilling efficiency in a percussive drilling system, while taking dynamic drill bit-rock interactions resulting from the piston impact into consideration. Drilling dynamic models (i.e., dynamic models of the drill bit) were established by introducing drill bit-rock interactions to determine the rock-penetrating properties of the bit. This was done using impact stress wave propagation theory, the penetration resistance coefficient, and the unloading constant in relation to the impacting piston and drill bit. The drilling dynamics was established considering the movement of the drill bit induced by piston impacts and the loading and unloading conditions resulting from the effects of the rock.

The drilling dynamics proposed here employ two dimensionless parameters: the first $(\alpha)$ is related to the pistonto-drill bit mass ratio and $\beta$ (defined above) and the second is $\beta$ itself. This introduction is followed by a discussion of the rate at which the impact energy is transmitted from the piston to the rock during percussion (i.e., the drilling efficiency). The drilling efficiency is the work completed by the drill bit relative to the incident-stress wave that causes it to crush the rock. We also analyzed the dynamics of the drill bit with regard to dimensionless parameters and damping ratios and considered impact-energy transmission rates given $m_{r}$ (the piston-to-drill bit mass ratio) and $\beta$. Combinations of parameters to maximize the efficiency of a percussive drilling system are proposed for various rock strengths.

To that end, Section 2 of this paper proposes a drilling dynamic model for a percussive drilling system that takes into account the dynamic properties of the drill bit, and with consideration to the theory of stress-wave propagation via piston impact. Drill bit behaviors and response characteristics observed during the percussion process (i.e., over, critical, and underdamped modes) involving the piston, the bit, and the rock via the parameters $\alpha$ and $\beta$ are discussed. The physical meaning was analyzed by vertical development of dimensionless parameters (i.e., $\alpha$ and $\beta$ ). The system responses of drill bit to the piston percussion were extended to six conditions depending on the presence of incident stress wave and loading or unloading condition. The typical motion of drill bits involves four conditions. However, this study expanded them into six conditions. The response characteristics resulting from the rock and the movement of the drill bit were also analyzed to investigate the efficiency or the transfer rate of the percussive energy from the piston to rock. 
The energy transmission efficiency via the impact of the percussive drilling system is examined in Section 3. Section 4 discusses the combinations of parameters that might optimize the drilling efficiency given the dynamic drill bit characteristics and rock stiffness (i.e., $\beta$ ). Conclusions are presented in Section 5.

\section{Dynamic Model of a Percussive Drilling System}

2.1. Mechanism of Percussive Drilling. Figures $1(\mathrm{a})$ and $1(\mathrm{~b})$ illustrate the working principles, or drilling mechanisms, of percussive drilling systems. In each case, the drill bit is propelled into the rock by percussion, feed, and rotation forces that are transmitted via the drifter or hammer. After receiving impact energy from the drill bit, the rock develops cracks that form a network and eventually break it. The crushed rock then undergoes further splitting via the rotation force and secondary impacts until it becomes powder. The rock powder is discharged to the outside by compressed air supplied through a flushing duct located inside the drill bit, thus enabling the drill to proceed further [1].

\subsection{Bit Motion and Stress Wave Propagation. Figure 2 il-} lustrates the principles of impact and rock penetration by a percussive drilling system.

The piston mass, length, cross-sectional area, descending speed, density, and longitudinal wave velocity are designated as $m_{p}, L_{p}, A_{p}, v, \rho$, and $c$, respectively. The underlying assumptions were that the drill bit remains immobile on the surface and that the diameters of the drill bit and the piston are equal. Also, the effects of buttons embedded in the bit were ignored.

The following equation expresses the motion of the drill bit resulting from the force applied to it by the piston [12]:

$$
m_{b} \frac{d^{2} u}{d t^{2}}=A\left(\sigma_{i}+\sigma_{r}\right)-F,
$$

where $u$ is the penetration depth (displacement) of the drill bit, the second term is the force applied to the drill bit when the impact is exerted upon it, $F$ is the force generated at the rock-bit interface (i.e., the penetration force), and $A$ is the cross-sectional area of the drill bit and piston.

According to the relation between the dynamic stress and velocity of a mass in an elastic body system, the velocity of the contacting end of the bit can be expressed as follows [2]:

$$
\frac{d u}{d t}=\frac{\sigma_{i}}{\rho c}-\frac{\sigma_{r}}{\rho c}
$$

here $\sigma_{i}$ and $\sigma_{r}$ are the incident and reflected stress waves, respectively. The effect of the secondary incident stress wave was not considered. For analytical simplification, the piston and the drill bit were assumed to be of the same diameter and material; both were assumed to have a rectangular pulse with a duration of incident stress wave $\tau$ and an amplitude of $0.5 \rho \mathrm{cv}$.

Typically, nearly all the percussion impact energy is converted into wave energy. The piston hitting the drill bit generates an incident stress wave $\left(\sigma_{i}\right)$, at which point a portion of the

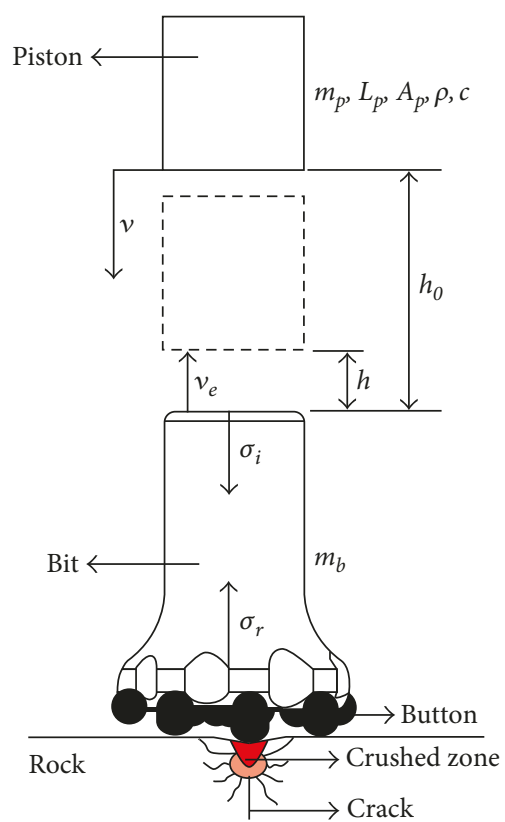

FIgURE 2: Schematic rock fracture mechanism by percussive drilling (modified from Cho et al. [13]).

impact energy is transmitted to the rock via the drill bit, crushing the rock [11]. The remaining energy is converted into a reflected stress wave $\left(\sigma_{r}\right)$ and is used to increase the speed of the piston ascending from the drill bit top to speed $v_{e}$ (Figure 2) [2]. The reflected stress wave can in turn be transmitted to the piston and drill bit in the form of a secondary incident stress wave at the piston-bit interface and at the drill bit-rock interface.

The secondary incident stress wave in a percussive drilling system has been reported not to contribute to the crushing of rocks [11]; therefore, it would not significantly affect the energy transmission efficiency. Furthermore, a study of drilling speed found the incident stress wave to have an insignificant effect on the impact force and penetration characteristics [14].

The interaction between the drill bit and rock (the force-penetration relationship, Figure 3) is expressed in (3) [2], which separately considers the loading and unloading conditions: $k$ is the rock penetration coefficient index, $\gamma$ is the unloading constant, and $\tau$ is the duration of the incident stress wave:

$$
F=\left\{\begin{array}{l}
k u \text { (loading), } \\
\gamma k u-\gamma k u_{f} \text { (unloading). }
\end{array}\right.
$$

The duration of the incident stress wave $\tau$ generated by the impact of the piston can be expressed with respect to time $t$ in two scenarios, as defined in (4) [2]. The first scenario involves transmission of an incident stress wave, whereas the second does not:

$$
\sigma_{i}(t)= \begin{cases}\frac{1}{2} \rho c v, & \text { for }(0<t \leq \tau), \\ 0, & \text { for }(t>\tau) .\end{cases}
$$




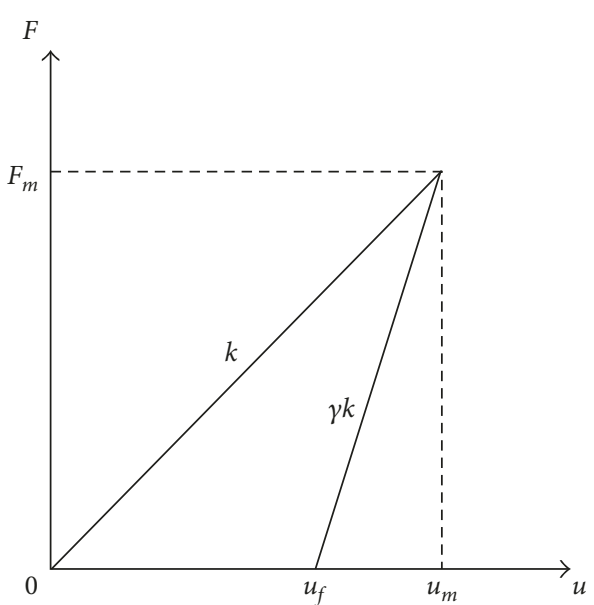

Figure 3: Force versus penetration relationship representing the bit-rock interaction curve.

For the loading condition, we obtain the following equations [2]:

$$
\begin{aligned}
A\left(\sigma_{i}+\sigma_{r}\right) & =F, \\
\frac{d u}{d t} & =\frac{\sigma_{i}}{\rho c}-\frac{\sigma_{r}}{\rho c}, \\
k u & =F,
\end{aligned}
$$

where the initial condition is $t=0$ and $F=0$.

For the unloading condition, the equations can be expressed as follows [2]:

$$
\begin{aligned}
A\left(\sigma_{i}+\sigma_{r}\right) & =F, \\
\frac{d u}{d t} & =\frac{\sigma_{i}}{\rho c}-\frac{\sigma_{r}}{\rho c}, \\
\gamma k u-\gamma k u & =F,
\end{aligned}
$$

where the initial condition is $t=\tau$ and $F(t)=\lim _{t \rightarrow \tau} F(t)$.

The response characteristics of a drill bit (i.e., the damped characteristics) can be under, critical, or overdamped given its motion equation, that is, (1). The movement of the drill bit under loading and unloading conditions was examined. The damping characteristics of a percussive drilling system are categorized depending on the damping ratio $(\zeta)$ and the dimensionless parameter $\alpha$.

When loading, the equation of motion for the drill bit is defined as follows:

$$
\ddot{u}+\left(\frac{\rho c A}{m_{b}}\right) \dot{u}+\left(\frac{k}{m_{b}}\right) u=\left(\frac{2 A}{m_{b}}\right) \sigma_{i},
$$

where the response-characteristics determinant $\zeta_{1}$ is

$$
\zeta_{1}=\frac{4 / \alpha(\beta / \tau)}{2 \sqrt{4 / \alpha(\beta / \tau)^{2}}}=\frac{1}{\sqrt{\alpha}} .
$$

When unloading, the drill-bit equation of motion is defined by (9). The $\zeta_{2}$ term is given in (10):

$$
\begin{aligned}
\ddot{u}+\left(\frac{\rho c A}{m_{b}}\right) \dot{u}+\gamma\left(\frac{k}{m_{b}}\right) u & =\left(\frac{2 A}{m_{b}}\right) \sigma_{i}+\gamma \frac{k u_{f}}{m_{b}}, \\
\zeta_{2} & =\frac{4 / \alpha(\beta / \tau)}{2 \sqrt{\gamma(4 / \alpha)(\beta / \tau)^{2}}}=\frac{1}{\sqrt{\gamma \alpha}} .
\end{aligned}
$$

The dimensionless parameters $\alpha$ and $\beta$ defined in (8) and (10) can be expressed as follows (extended from (2), (12)):

$$
\begin{aligned}
& \alpha=\frac{4 m_{b}}{\rho c A} \cdot \frac{k}{\rho c A}=\frac{4 m_{b}}{\rho c A \tau} \cdot \beta=\frac{4 m_{b}}{2 m_{p} L_{P} / c L_{P} A \beta}=2\left(\frac{m_{b}}{m_{p}}\right) \beta, \\
& \beta=\frac{k \tau}{\rho c A}=\frac{k 2 L_{p} / c}{\rho c A}=\frac{2 k L_{h}}{\rho c^{2} A}=\frac{2 k L_{p}}{\rho A\left(E_{p} / \rho\right)}=2 k\left(\frac{L_{p}}{E_{p} A}\right)=2\left(\frac{k}{k_{p}}\right) .
\end{aligned}
$$

As explained earlier, the typical motion of drill bits involves four conditions. However, this study considers six conditions, as listed in Table 1 (Appendix A), and includes the loading/unloading condition and the presence or absence of an incident stress wave. To investigate the rate at which the impact energy is transmitted from the piston to the rock during the percussion process (i.e., the drilling efficiency), this study first considered the response characteristics resulting from the motion of the drill bit during the percussion process and the effect of the rock. The aim was to find the response characteristics that lead to the rock's maximum crushing displacement, $u_{m}$. The study also defined the motion of percussive drill bits resulting from the impact of the piston and analyzed their behavior given the system responses. The drill bit-rock interaction was considered here, including the force-penetration relationship for the rock.

Table 1 presents the relationships between loading and unloading and the duration of stress wave $\tau$ in percussive drilling systems. In Table 1, $\tau$ is the duration of the incident stress wave being transmitted to the drill bit via the piston during loading; it is referenced relative to the time $t$. The loading condition can be defined in two ways: $0<t \leq \tau$ or $\tau<t \leq t_{m}$, where the latter expression means that $\tau$ arrived sooner than $t$; that is, the transmission of $\tau$ was complete, but the loading continued owing to the inertial effect of the drill bit. If $\tau>t_{m}$, the drill bit arrived at $u_{m}$ before the transmission of $\tau$ to the bit had completed and indicates the time when the system switches to the unloading condition.

In summary, in Cases 1 to 5 in Table 1 , the motion of the drill bit following the piston impact continues until the loading condition applying external force to the rock reaches $\tau<t \leq t_{m}$. After that time, the stress wave arriving at the drill bit is converted into unloading at $\tau>t_{m}$, provided that the rock displacement occurs sooner than $t_{m}$, that is, the maximum time of arrival. However, the $\tau$ in Case 6 represents a stress wave that is still progressing while the rock displacement owing to the drill bit has already reached its maximum; that is, unloading occurs during $\tau$. 
TABLE 1: Expansion conditions for dynamic drill-bit properties for loading, unloading, and stress-wave duration, $\tau$.

\begin{tabular}{|c|c|c|c|}
\hline \multirow[b]{2}{*}{ Case no. } & \multicolumn{3}{|c|}{ Initial conditions } \\
\hline & $\begin{array}{c}0<t \leq \tau \\
\sigma_{i}=0.5 \rho c v \\
F=k u \\
u_{0}=0, \dot{u}_{0}=0\end{array}$ & $\begin{array}{c}\tau<t \leq t_{m} \\
\sigma_{i}=0 \\
F=k u \\
u_{0}=u(\tau), \dot{u}_{0}=\dot{u}(\tau)\end{array}$ & $\begin{array}{c}t>t_{m} \\
\sigma_{i}=0 \\
F=\gamma k u-\gamma k u_{f} \\
u_{0}=u_{m}, \dot{u}_{0}=0\end{array}$ \\
\hline 1 & & $\alpha<\frac{1}{\gamma}$ & \\
\hline 2 & & $\alpha=\frac{1}{\gamma}$ & \\
\hline 3 & & $\frac{1}{\gamma}<\alpha<1$ & \\
\hline 4 & & $\alpha=1$ & \\
\hline 5 & & $\alpha>1, t_{m}>\tau$ & \\
\hline & $0<t \leq t_{m}$ & $t_{m}<t \leq \tau$ & $t>\tau$ \\
\hline 6 & $\begin{array}{c}\sigma_{i}=0.5 \rho c v \\
F=k u \\
u_{0}=0, \dot{u}_{0}=0\end{array}$ & $\begin{array}{c}\sigma_{i}=0.5 \rho c v \\
F=\gamma k u-\gamma k u_{f} \\
u_{0}=u_{m}, \dot{u}_{0}=0\end{array}$ & $\begin{array}{c}\sigma_{i}=0 \\
F=F=\gamma k u-\gamma k u_{f} \\
u_{0}=u(\tau), \dot{u}_{0}=\dot{u}(\tau)\end{array}$ \\
\hline
\end{tabular}

2.3. Results of Bit Motion Analysis. Numerical simulations on a dynamic model of drill bit properties were run for the six cases and used the dynamic drill bit properties during induced percussion (Section 2), the drill bit-rock interaction model, and the dimensionless parameters $\alpha$ and $\beta$.

Figure 4 shows the simulation results $(\zeta)$ for different conditions ( $\alpha$, Table 1) and the effect of loading and unloading. The mass ratio, $m_{r}$, was 1 , and the same conditions were applied to the piston mass and drill bit mass. Figure 4(a) shows an overview of the response characteristics for all six cases. The initial black part of each curve refers to the sustained effect of $\tau$; the red parts indicate the loading condition that marks the effect of drill bit inertia, and the blue parts represent the transition to unloading. Figure 4(b) shows Case 1, where a single-incident stress wave indicates the drill bit displacement at $u(\tau)$ and the drill bit arrival at $u_{m}$ as an overdamped motion due to the loading continuing owing to external forces and inertia. Later, in the transition to unloading, characteristic overdamped motion appears, and there is no convergence to $u_{f}$ (Figure 3).

Case 2 (Figure 4(c)) appears similar to Case 1, but the bit reaches $u_{m}$ sooner owing to $\tau$ and the effect of drill bit inertia. As the system transitions to unloading and converges to $u_{f}$, it shows critical damping. Case 3 (Figure 4(d)) shows a longer $\tau$ compared with Case 2 because of its quicker arrival at $u_{m}$. During unloading, it converges to $u_{f}$ as underdamped motion. Case 4 (Figure $4(\mathrm{e})$ ) has the drill bit displaced at $u(\tau)$ due to $\tau$, and it arrives at $u_{m}$ as critically damped motion owing to continued loading resulting from the inertia of the drill bit. The subsequent unloading phase converges to $u_{f}$ as underdamped motion.

Case 5 (Figure 4(f)) has a longer $\tau$ than the preceding four cases and also reaches $u_{m}$ sooner. Furthermore, all its zones showed underdamped motion. Finally, in Case 6 (Figure 4 (g)), the rock displacement has already reached its maximum while $\tau$ is still in progress. This means that unloading begins during $\tau$. Underdamped motion was observed in all zones.

Overall, the longer the duration of $\tau$, the shorter the loading time and the sooner the $u_{m}$ is reached, owing to drill bit inertia. Table 2 summarizes the damping characteristics for the loading and unloading conditions and presents the associated damping mode that arrives at $u_{m}$. Cases 1-3 show overdamping, Case 4 critical damping, and Cases 5 and 6 underdamping. The underdamped mode showed the highest responsiveness, indicating the fastest transmission time of $\tau$. The results show that increasing the $\tau$-transmission time decreased the loading effect resulting from drill bit inertia and led to faster responses. The drill bit inertial effect is inversely proportional to $\tau$.

\section{Energy Transmission in Percussive Drilling System}

In Section 2, we examined the response characteristics of drill bits that can arrive at $u_{m}$, which is the maximum drilling displacement of rocks. Based on the examined characteristics in Section 3, we calculate the efficiency of percussive drilling systems and discuss the combinations of parameters that could maximize it.

3.1. Energy Transmission Efficiency. In a percussive drilling system, the piston directly hits the drill bit at speed $v$ (Figure 2). For a transmitted impact energy, $E_{i}$, at the time of the piston descending and hitting the drill bit, the final speed of the piston immediately preceding the impact, $v$, can be expressed as follows [2, 10]:

$$
v=\left(\frac{2 E_{i}}{m_{p}}\right)^{1 / 2} .
$$



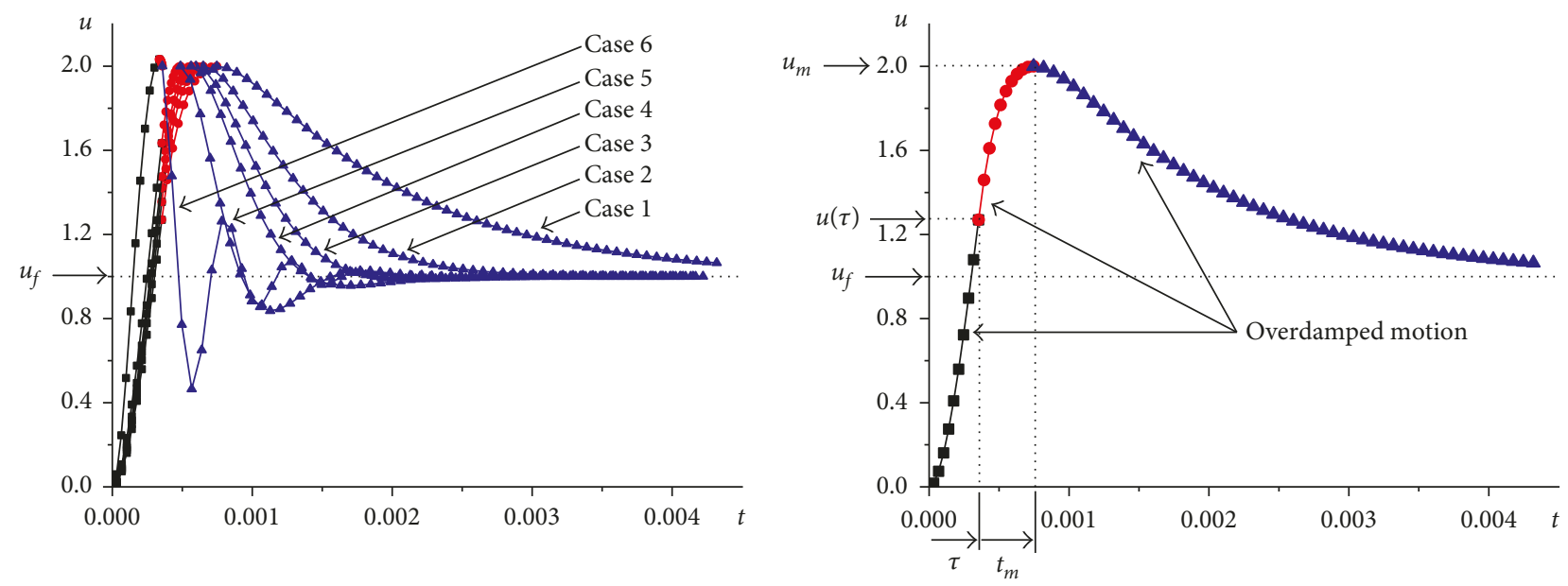

- Loading by incident stress wave

$\rightarrow$ Loading by incident stress wave

- - Loading by inertia of bit

$\rightarrow$ Unloading

- - Loading by inertia of bit

$\rightarrow$ Unloading

(a)

(b)

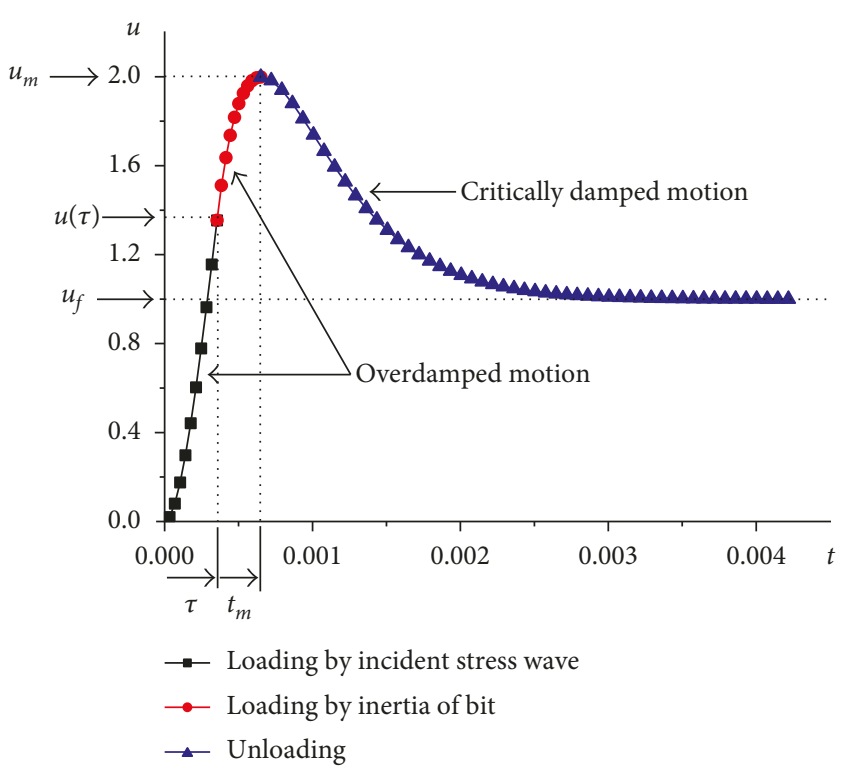

(c)

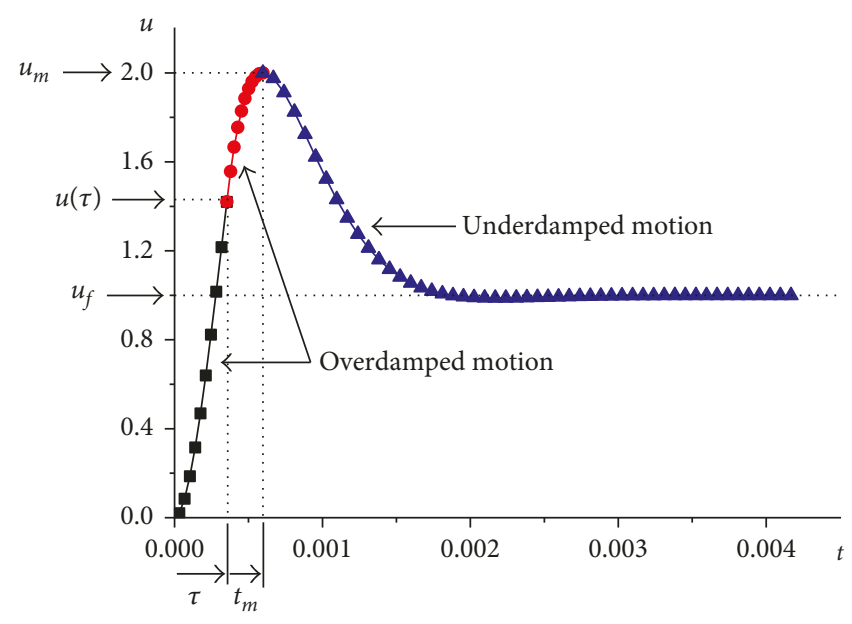

$\rightarrow$ Loading by incident stress wave

- - Loading by inertia of bit

$\leadsto$ Unloading

(d)

FIgUre 4: Continued. 

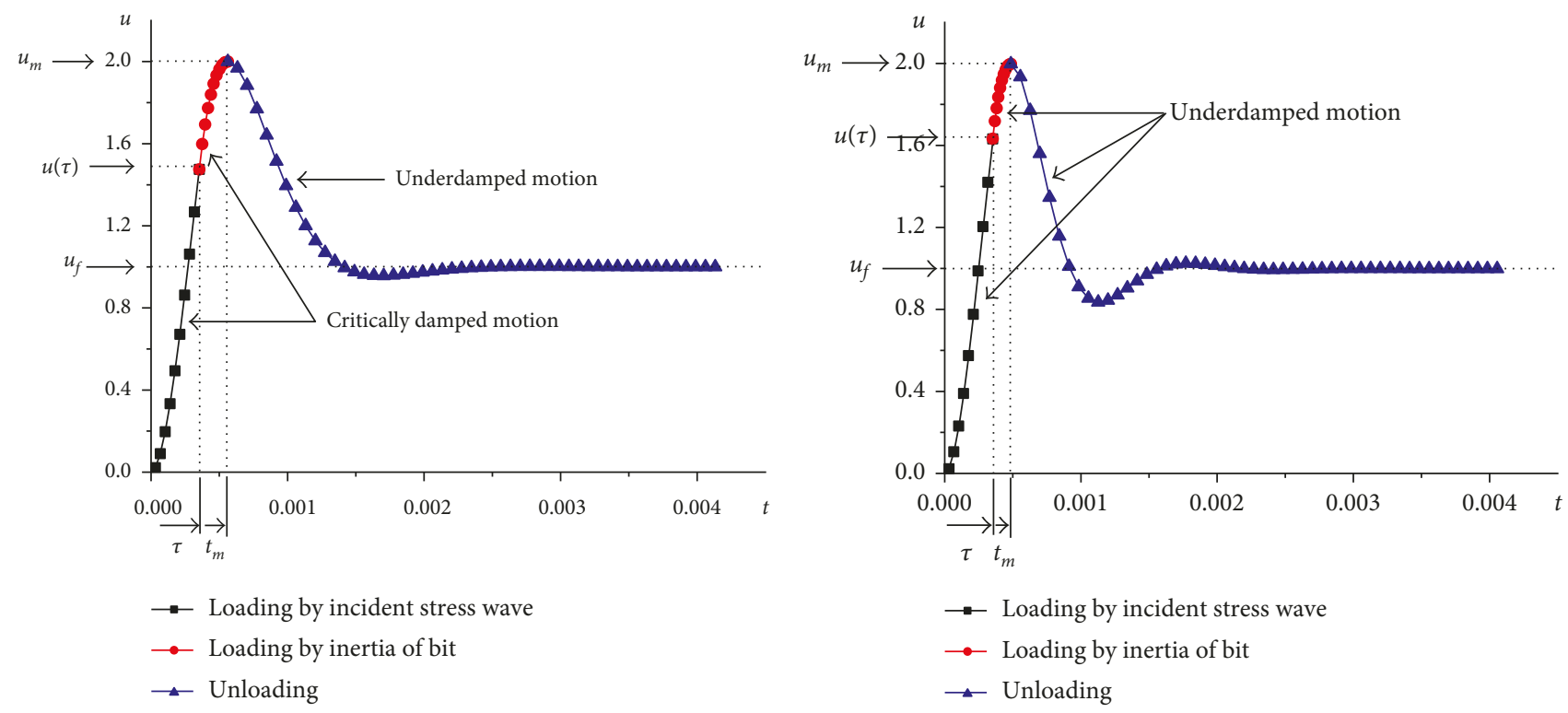

(e)

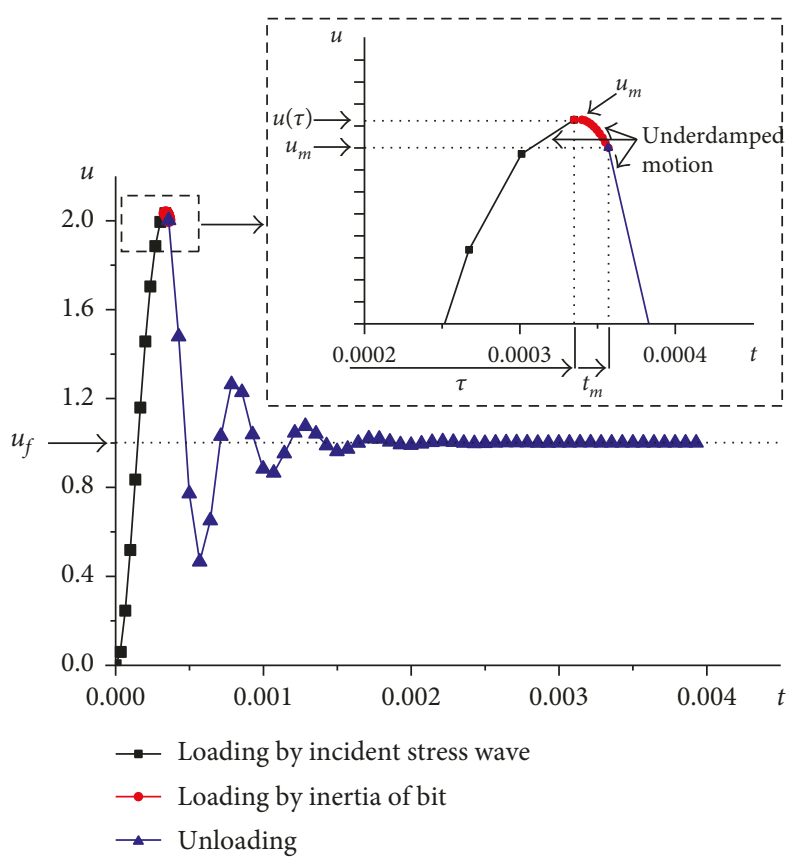

(g)

Figure 4: Results of the motion response of the bit for $m_{r}=1$. (a) All cases. (b) Case 1. (c) Case 2. (d) Case 3. (e) Case 4. (f) Case 5. (g) Case 6.

The initial height $h_{0}$ is $v^{2} / 2 g$, and the rebound, $e_{h}$, can be defined as the ratio between the initial height and the piston's postimpact rebound height, $h$. These are expressed as follows [2]:

$$
e_{h}=\frac{h}{h_{0}}=\frac{v_{e}^{2}}{v^{2}}
$$

The efficiency, $\eta$, of impact energy transmission is defined as the ratio between the kinetic energy generated from the impact of the piston and the energy transmitted to the rock [12] and is given as

$$
\eta=\frac{k u_{m}{ }^{2}}{\rho A L_{h} v^{2}}=\frac{\left(F_{\max }\right)^{2}}{\rho A L_{h} v^{2} k},
$$

Which can be expressed as follows through dimensional analysis:

$$
\eta=\frac{2}{\beta} u_{m}^{2}
$$

The value $u_{m}$ during loading can be obtained from (7), the equation of motion for the drill bit. The obtained $u_{m}$ can be expressed as in (16) for each damping mode, where the initial conditions are $0<t \leq \tau, F=k u_{i}, u_{0}=0$, and $\dot{u}_{0}=0$ : 
TABLE 2: Dynamic drill-bit properties for loading, unloading, and $\tau$, and the damping modes capable of reaching $u_{m}$.

\begin{tabular}{|c|c|c|c|c|}
\hline \multirow{2}{*}{ Case no. } & \multirow{2}{*}{ Conditions } & \multicolumn{3}{|c|}{ Damping modes capable of reaching $u_{m}$} \\
\hline & & $0<t \leq \tau$ & $\tau<t \leq t_{m}$ & $t>t_{m}$ \\
\hline 1 & $\alpha<\frac{1}{\gamma}$ & Over & Over* & Over \\
\hline 2 & $\alpha=\frac{1}{\gamma}$ & Over & Over* & Critical \\
\hline 3 & $\frac{1}{\gamma}<\alpha<1$ & Over & Over* & Under \\
\hline 4 & $\alpha=1$ & Critical & Critical* & Under \\
\hline 5 & $\alpha>1, t_{m}>\tau$ & Under & Under* & Under \\
\hline 6 & $\alpha>1, t_{m}<\tau$ & $\begin{array}{c}0<t \leq t_{m} \\
\text { Under* }^{*}\end{array}$ & $\begin{array}{c}t_{m}<t \leq \tau \\
\text { Under }\end{array}$ & $\begin{array}{c}t>\tau \\
\text { Under }\end{array}$ \\
\hline
\end{tabular}

* Damping modes capable of reaching $u_{m}$.

$$
u_{m}(t)\left\{\begin{array}{l}
1+\left[\frac{1-\sqrt{\alpha-1}}{2 \sqrt{\alpha-1}} \cdot e^{-(2 / \alpha)(\beta / \tau)(1+\sqrt{1-\alpha}) t}-\frac{1+\sqrt{1-\alpha}}{2 \sqrt{1-\alpha}} \cdot e^{-(2 / \alpha)(\beta / \tau)(1-\sqrt{1-\alpha}) t}\right] \text { (overdamped), } \\
1-e^{-2(\beta / \tau) t} \cdot\left[2 \frac{\beta}{\tau} t+1\right](\text { critical damped), } \\
1-e^{-2(\beta / \tau) t} \cdot\left[\frac{1}{\sqrt{\alpha-1}} \sin \left(\frac{2 \sqrt{\alpha-1}}{\alpha} \frac{\beta}{\tau} t\right)+\cos \left(\frac{2 \sqrt{\alpha-1}}{\alpha} \frac{\beta}{\tau} t\right)\right] \text { (underdamped). }
\end{array}\right.
$$

Moreover, for $t_{m}<\tau, u_{m}$ is expressed as follows:

$$
\begin{aligned}
u_{m}(t)= & 1-e^{-(2 / \alpha)(\beta / \tau)} \cdot\left[\frac{1}{\sqrt{\alpha-1}} \sin \left(\frac{2 \sqrt{\alpha-1}}{\alpha} \frac{\beta}{\tau} t\right)\right. \\
& \left.+\cos \left(\frac{2 \sqrt{\alpha-1}}{\alpha} \frac{\beta}{\tau} t\right)\right] .
\end{aligned}
$$

The initial conditions are $0<t \leq \tau, F=k u_{i}, u_{i}=0$, and $\dot{u}_{i}=0$.

Next, the displacement value $u_{f}$ during unloading can be obtained via the equation of motion for a drillbit (9), as shown in (18). The initial conditions during unloading are $t>\tau, \quad F=0, \quad u_{0}=u(\tau)$, and $\dot{u}_{0}=\dot{u}(\tau)$ :

$$
u_{f}(t)\left\{\begin{array}{l}
\frac{\alpha}{4 \sqrt{1-\alpha}} \frac{\tau}{\beta} \cdot e^{-(2 / \alpha)(1+\sqrt{1-\alpha}) t} \cdot\left[u_{0} \frac{2}{\alpha} \frac{\beta}{\tau}(1+\sqrt{1-\alpha})-\left(\dot{u}_{0}+\frac{4}{\alpha} \frac{\beta}{\tau} u_{0}\right)\right] \\
-\frac{\alpha}{4 \sqrt{1-\alpha}} \frac{\tau}{\beta} \cdot e^{-(2 / \alpha)(1-\sqrt{1-\alpha}) t} \cdot\left[u_{0} \frac{2}{\alpha} \frac{\beta}{\tau}(1-\sqrt{1-\alpha})-\left(\dot{u}_{0}+\frac{4}{\alpha} \frac{\beta}{\tau} u_{0}\right)\right](\text { overdamped }), \\
e^{-2(\beta / \tau) t} \cdot\left[\left(\dot{u}_{0}+2 \frac{\beta}{\tau} u_{0}\right) t-u_{0}\right](\operatorname{critical~damped}), \\
e^{-(2 / \alpha)(\beta / \tau) t} \cdot\left[\left(\frac{\alpha}{2 \sqrt{\alpha-1}} \frac{\tau}{\beta}\right) \dot{u}_{0} \cdot \sin \left(\frac{2 \sqrt{\alpha-1}}{\alpha}\right) \frac{\beta}{\tau} t+\frac{1}{\sqrt{\alpha-1}} u_{0} \cdot \sin \left(\frac{2 \sqrt{\alpha-1}}{\alpha} t\right)\right. \\
\left.+u_{0} \cdot \cos \left(\frac{2 \sqrt{\alpha-1}}{\alpha} \frac{\beta}{\tau} t\right)\right](\text { underdamped }) .
\end{array}\right.
$$




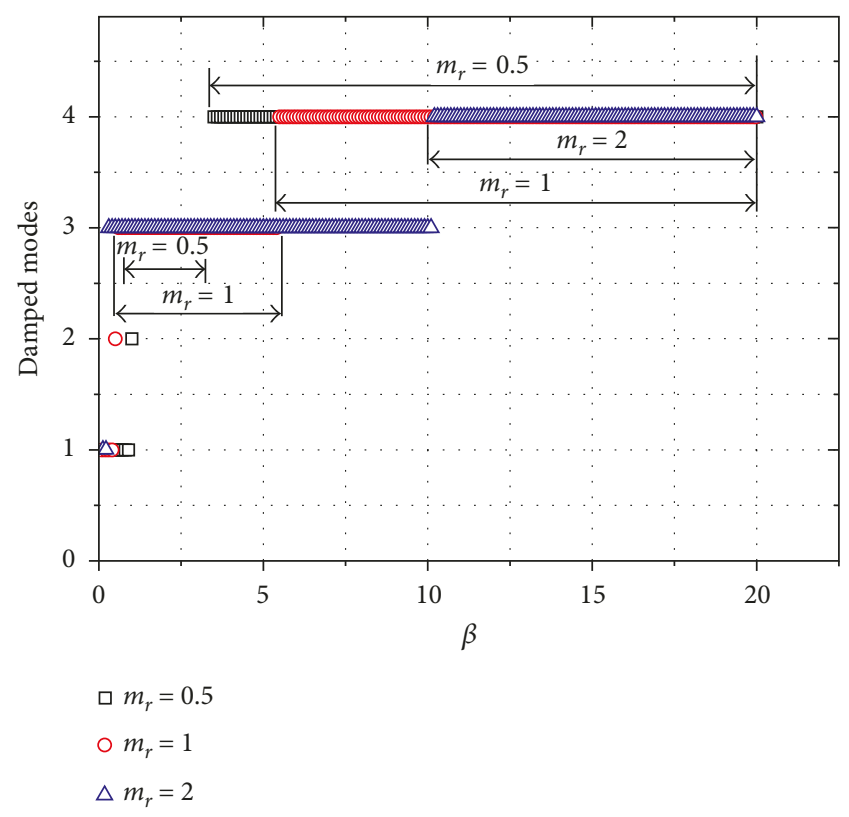

FIGURE 5: Damping mode capable of reaching maximum rock fracture displacement $u_{m}$ given the piston-to-bit mass ratio $m_{r}$ and $\beta$. 1: overdamped mode, 2 : critically damped mode, 3 : underdamped mode, 4: special underdamped mode.

The efficiency of the percussive drilling systems for given $\beta$ can be calculated using (14-17). The factor $u^{m}$, which is important to the efficiency calculations, is discussed in depth.

3.2. Results of Energy Transmission Efficiency. The results of simulations on the drill bit-rock interaction model shown in Figure 5 indicate the damped modes that can arrive at $u_{m}$ (for varying values of $\beta$ ) as the response by the drill bit at the time of piston impact. The damped mode is determined by $\zeta$ and $\alpha$, which indictate the system responses. Overdamped mode has $\alpha<1$ and can be expressed as follows:

$$
\begin{aligned}
2\left(\frac{m_{b}}{m_{h}}\right) \beta & <1, \\
\beta & <\frac{1}{2 m_{r}} .
\end{aligned}
$$
follows:

The critical damped mode has $\alpha=1$ and is expressed as

$$
\begin{aligned}
2\left(\frac{m_{b}}{m_{h}}\right) \beta & =1, \\
\beta & =\frac{1}{2 m_{r}} .
\end{aligned}
$$

The underdamped mode has $\alpha>1$ and is expressed as

$$
\begin{aligned}
2\left(\frac{m_{b}}{m_{h}}\right) \beta & >1, \\
\beta & >\frac{1}{2 m_{r}},
\end{aligned}
$$

where $m_{b} / m_{h}=m_{r}$.

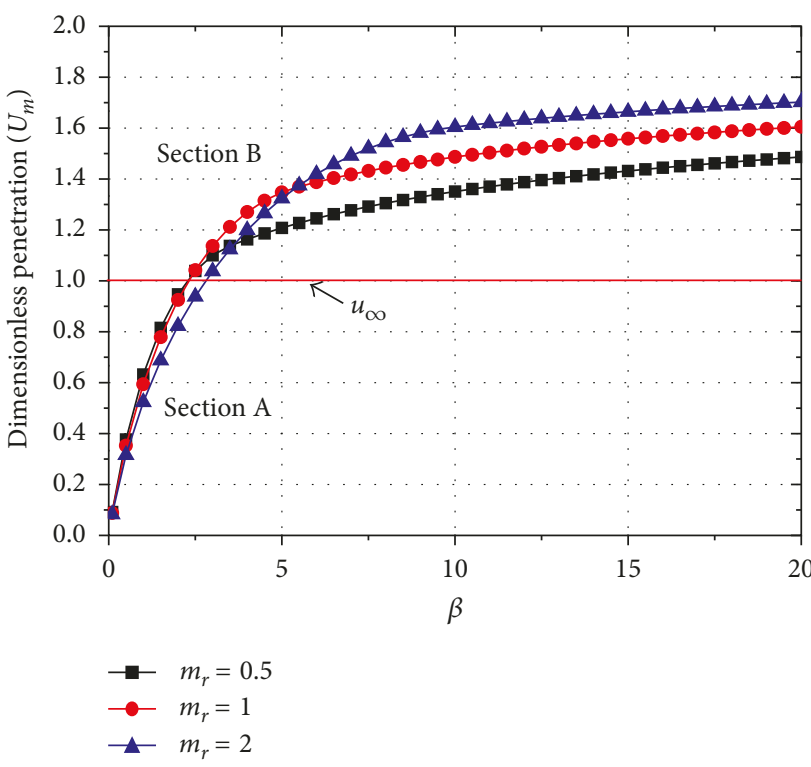

FIgURE 6: State of reaching drill bit dimensionless penetration $U_{m}$ with respect to mass ratio, $m_{r}$, and $\beta$.

The special underdamped mode refers to the case where $\tau$ is faster than $t$, indicating that the drill bit can arrive at $u_{m}$ only via an incident stress wave. In this case, the bit reaches its maximum displacement and transitions to the unloading state while the transmission of $\tau$ is underway.

The damped mode of each $m_{r}$ shown in Figure 5 reveals that the underdamped mode is dominant of the effects. Also, overdamped and critical damped were observed, where $\beta$ was low. This is possibly because of the effects of the internal energy (i.e., compression strength) of rock and the percussive energy. The overdamped and critical damped were also observed. This is possibly because of internal energy (i.e., compression strength) of rock and energy caused by percussion.

Figure 6 shows dimensionless time, $t_{m}=t / \tau$, capable of reaching $U_{m}$ for $\beta$ and $\tau$. In Section A of $t>\tau$, the bit reaches $U_{m}$ after termination of $\tau$, and in Section B of $t<\tau$, the bit reaches $U_{m}$ before the termination of $\tau$. The lower the $m_{r}$ and $\beta$, the faster the termination of $\tau$, and increase in these tended to make the duration of $\tau$ delivered to rock through bit to increase proportionally to $\beta$. This suggests that the higher rock strength requires longer duration of $\tau$ for drilling the rock.

Figure 7 shows dimensionless $U_{m}$ state defined as ratio $u_{m}$, a displacement $u_{m}$ by $\beta$, and $u_{\infty}$ caused by incident stress wave and loading condition. The prediction of displacement of drill bit, drilling displacement, by characteristic of rock was limited. This study, accordingly, judged the dimensionless drilling state, $U_{m}$, only by using ratio $u_{\infty}$, a displacement during loading and $u_{m}$ considering inertia caused during percussion (22):

$$
\bar{U}_{m}=\frac{u_{m}}{u_{\infty}},
$$

where the $u_{\infty}$ only by loading is defined as follows: 


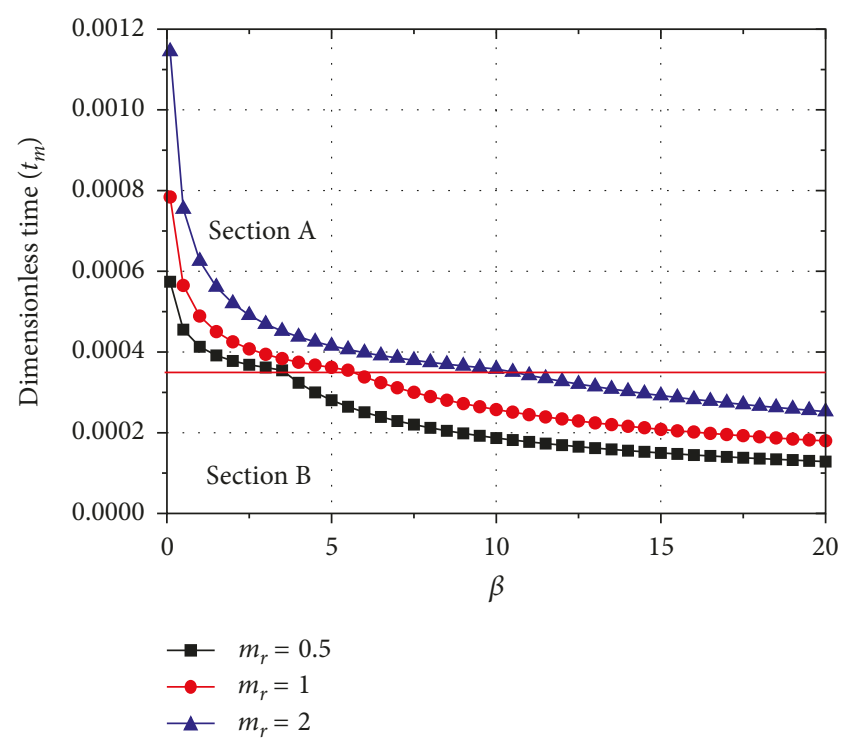

Figure 7: Dimensionless time capable of reaching $U_{m}$ for an incident stress-wave $\tau$ acting on the bit and $\beta$.

$$
u_{\infty}=\frac{\rho c A}{k} v=\frac{\tau}{\beta} v
$$

The effect of initial condition on $u_{\infty}$ decreased with the increase in $\beta$. The $u_{m}$ is determined by considering loading condition and inertia effect, is affected by overresponse, and decreases as $\beta$ increases.

Section A refers to $u_{m}<u_{\infty}$, with only $\tau$ and loading effect present (i.e., the condition for the normal state in the initial mode): $u_{\infty}$ means only the $\tau$ effect resulting from loading exists. Section B refers to $u_{m}>u_{\infty}$, a state in which $u_{m}$ can be reached through $\tau$ and loading is exceeded. This section is where the drill bit inertia and underdamped motion appear. The gradient of the curve for maximum displacement of the bit varies with $\beta$, indicating that as the rock strength increases, the displacement allowing fracture decreases. Near value 2, $\beta$ changes its gradient for maximum displacement. This means that, in Section A, the rock is less stiff than the piston owing to the rock-stiffness effect, and in Section $B$, the rock is stiffer than the piston. $\beta$ is defined as the stiffness ratio between the rock and the piston, as in (11). Hence, when $\beta<2$, a condition is established for rock fracture to occur, in that the piston stiffness exceeds the rock stiffness.

In examining $u_{m}$ for piston-bit mass ratios of $m_{r}<2$, the rock strength that can be defeated by $\tau$ occurring in the initial loading tends to increase. This tendency is suspected to result from the inertial effect of the mass of the bit. For $\beta>4$, the $u_{m}$ gradient tended to decrease with increasing mass of the bit. Hence, the efficiency is considered to decrease, which is attributed to the effect of the rock's compressive strength. The uniaxial compressive strength of very hard rock is typically at least $200 \mathrm{MPa}$, while the tensile strength of the H13 tool steels that are used mostly for pistons (ASTM A681 and DIN EN ISO 4957) is known to be at least 359-1170 MPa [15].

Given the steels generally used to make pistons, the effective range of $\beta$ is considered to be $0.34<\beta<1.12$, which

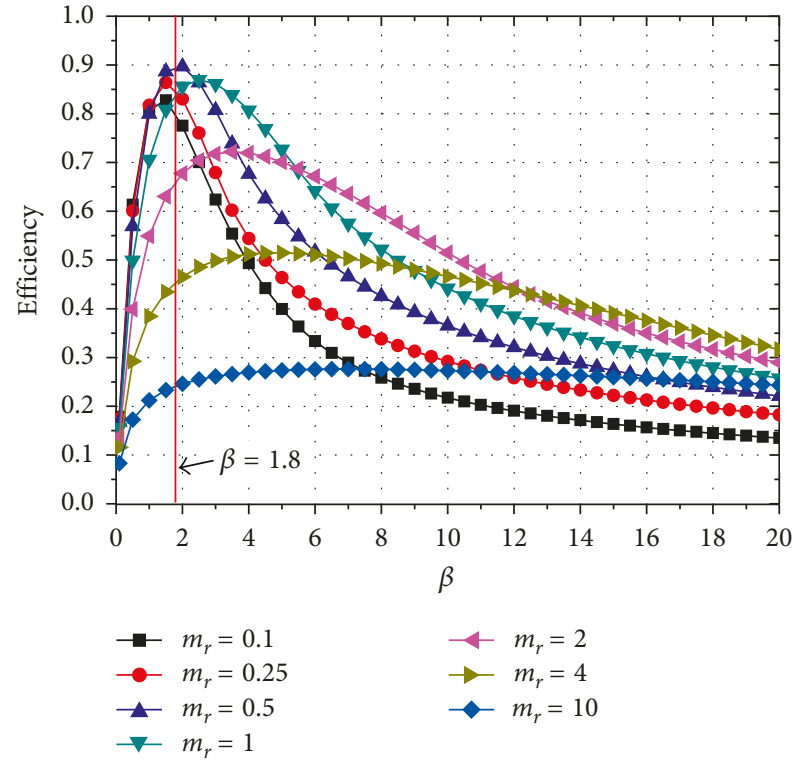

FIgURE 8: Drilling efficiency of percussive drilling systems with respect to $\beta$ and $m_{r}$.

was calculated considering the strength ratio between the hard rock and an $\mathrm{H} 13$ steel tool. Hence, the effective range of $\beta$ could be no greater than 2 . Rocks with a stiffness ratio of $\beta>2$ might cause failure through the piston's plastic deformation and fracture. This study examined the effective interval of $\beta$ and investigated the parameter combinations giving high drilling efficiency in this interval.

Figure 8 illustrates the motion of the bit generated during the percussion process and the energy transmission rate (i.e., efficiency) for the response characteristics, $\tau$, and percussion impact. Analysis of $m_{r}$ found the maximum drilling efficiency in interval $1<\beta<2$, with a tendency efficiency decreasing after $m_{r}$ increased above a certain value. This result was attributed to the tendency whereby increasing $m_{r}$ converts the system's dynamic response to $\tau$ due to the piston from underdamped to overdamped. Furthermore, increasing the mass of the bit can increase its internal energy (i.e., friction energy) to above the piston transmitted energy (i.e., kinetic and potential energy). Hence, the bit's dynamic response would be overdamped and could compromise drilling efficiency. In other words, the heavier the bit, the more delayed the response to percussion.

In Figure 8 , the left side of red line $(\beta=1.8)$ represents the effect of only incident stress wave, meaning that the higher the value in graph the higher the drilling efficiency in incident stress wave. The right side of red line, representing unloading behavior after reaching maximum efficiency in incident stress wave, is affected by both brittleness characteristics of rock and inertia effect of drill bit.

The piston should have twice the mass of the drill bit (i.e., $m_{r}=0.5$ ) for the most efficient drilling for $\beta \leq 2$, which is established to be the effective interval of $\beta$ and corresponds to both soft and hard rocks. Drilling stiffer rocks $(2<\beta<4)$ might be less efficient, but increasing $m_{r}$ to 1 would help improve drilling efficiency. 


\section{Results and Discussion}

This study defined equations for the motion of a drill bit when struck by a piston and expanded the dynamic properties of the drill bit based on the conditions $\zeta$ and $\alpha$. It also analyzed the relationship between the bit dynamics and the duration of the incident stress wave $(\tau)$. Underdamped drill bits displayed the greatest responsiveness and the fastest transmission of $\tau$. Increasing the transmission of $\tau$ decreased the loading effect due to the drill bit's inertia but led to a fast response. The inertial effect of the drill bit was confirmed to be inversely proportional to the transmission of $\tau$.

A percussive drilling system accomplishes its work via the percussion impact of a piston. In the systems studied here, $\tau$ ended more quickly with lower $m_{r}$ and $\beta$ values; as these values increased, the duration of $\tau$ transmitted to the rock via the drill bit increased in proportion to $\beta$. The results also showed that the maximum bit displacement $\left(u_{m}\right)$ decreased as $\beta$ increased. Analysis of the drilling dynamics confirmed the effective intervals of $\beta$. With reference to the rock strengths suggested by the International Society for Rock Mechanics (ISRM), the effective interval of $\beta$ is considered to be no greater than 2 .

The main purpose of this study was to examine the impact energy transmission rate and drilling efficiency in a percussive drilling system (Figure 8). The results established a $m_{r}$ value of 0.5 as the most efficient for rocks whose strength corresponds to the interval $\beta<2$. The improved drilling efficiency would lead to advantages such as reduced bit production costs. Rocks stiffer than $2<\beta<4$ can be most efficiently drilled by selecting $m_{r}=1$.

The movement and response characteristics of bit during percussion process, $\tau$, and transfer rate of energy caused by percussion, an efficiency, depend on $m_{r}$ and $\beta$, where increase in $m_{r}$ leads to overdamping of bit movement characteristics due to $\tau$ by piston and reduction in drilling efficiency due to internal energy effect by mass of drill bit. As the increase in mass of drill bit, for the section of $4<\beta<6$, the drilling efficiency of percussive drilling system decrease, and for the very hard rock (UCS $>200 \mathrm{MPa}$ ) section of $4<\beta<6$, it is considered that the reasonable applied $m_{r}$ of drilling tool is 1 or 2 .

This study neglected the effects of the secondary incident stress wave and also the effect of buttons embedded in the drill bit. Additionally, for analytical simplification, the piston and drill bit were assumed to be of the same diameter and material, and a rectangular pulse with an incident stress wave of duration $\tau$ and amplitude $0.5 \rho c v$ was assumed. The effect of the flexural stress wave depending on the configuration was not considered. These limitations of this paper should be addressed in further investigations that take into account the configuration of the piston and drill bit, the effect of buttons, and the drilling efficiency given differing shapes of the incident stress wave.

\section{Conclusion}

This paper aimed to identify the optimal design parameters for percussive drilling systems by introducing a drill bit-rock interaction model that could verify the bit's motion during percussion and the resulting damping characteristics. The study analyzed drilling efficiency and drew the following conclusions.

Percussive drilling systems have six dynamic drill bit properties that can be expandable. This paper discussed the physical meaning of the dimensionless parameters $\alpha$ and $\beta$. Their values determined the damping characteristics that can lead to the rock's maximum fracture displacement. The fastest response of $\tau$ was observed for underdamped drill-bit motion.

Drilling was most efficient in the interval $1<\beta<4$, where increasing $m_{r}$ at a given $\beta$ decreased drilling efficiency. The value of $m_{r}$ for efficient drilling was determined by the rock strength (i.e., $\beta$ ).

The results indicate that application of a piston-to-drill bit mass ratio of 0.5 (i.e., a piston mass twice that of the drill bit mass) to the rocks whose stiffness corresponds to $\beta \geq 2$ would likely be most efficient and also reduce drill bit production costs. Furthermore, a selection of $m_{r}=1$ would be valid when developing drill tools for boring complex rocks $(1<\beta<4)$. At $\beta>4$, the best efficiency could be achieved when the bit mass equals or exceeds the piston mass.

\section{Appendix}

\section{Theoretical Case of Bit Motion Condition (Drilling Dynamic Model)}

This paper proposed six conditions for the dynamics of a drill bit struck by a piston impact, as summarized below. The damping conditions for the damping ratios and $\alpha$ from the drill-bit equations of motion (7-9) are as follows:

First, for the overdamped condition of $\zeta_{1}>1$ and $\alpha<1, \tau$ is transmitted to the drill bit, indicating loading. Equation (7) can be expressed as in (A.1). The initial conditions are $0<t \leq \tau, \sigma_{i}=0.5 \rho c v, F=k u, u_{0}=0$, and $\dot{u}_{0}=0$.

$$
\ddot{u}+\left(\frac{\rho c A}{m_{b}}\right) \dot{u}+\left(\frac{k}{m_{b}}\right) u=\left(\frac{\rho c A}{m_{b}}\right) v,
$$

where assuming the normal state, $u$ can be calculated as follows:

$$
u=\left(\frac{\rho c A}{k}\right) v .
$$

Next, we examine the case where $\tau$ is complete and the loading effect is sustained owing to external forces and drillbit inertia. Applying the conditions of $\tau<t \leq t_{m}, \sigma_{i}=0$, $F=k u, u_{0}=u(\tau)$, and $\dot{u}_{0}=\dot{u}(\tau)$ to (A.1) results in the following expression:

$$
\ddot{u}+\left(\frac{\rho c A}{m_{b}}\right) \dot{u}+\left(\frac{k}{m_{b}}\right) u=0,
$$

where $u=0$. Last, we examine the unloading conditions of $t_{m}<t, \sigma_{i}=0, F=\gamma k u-\gamma k u_{f}, u_{0}=u_{m}$, and $\dot{u}_{0}=0$. Applying these to (9) when unloading gives 


$$
\ddot{u}+\left(\frac{\rho c A}{m_{b}}\right) \dot{u}+\gamma\left(\frac{k}{m_{b}}\right) u=\gamma\left(\frac{k}{m_{b}}\right) u_{f},
$$

where bit displacement $u=u_{f}$ is used to indicate the rock's fracture displacement. Furthermore, considering the bit's damping ratio when unloading, its behavior can be expressed as

$$
\text { Damped mode }= \begin{cases}\zeta_{2}>1, & \alpha<\frac{1}{\gamma} \text { (over), } \\ \zeta_{2}=1, & \alpha=\frac{1}{\gamma} \text { (critical), } \\ \zeta_{2}<1, & \alpha>\frac{1}{\gamma} \text { (under). }\end{cases}
$$

Second, under critical damping where $\zeta_{1}=1$ and $\alpha=1, \tau$ is transmitted to the drill bit, indicating loading. Equation (7) can be expressed as in (A.1). The initial conditions are $0<t \leq \tau, \sigma_{i}=0.5 \rho c v, F=k u, u_{0}=0$, and $\dot{u}_{0}=0$.

In this case, assuming the normal state, $u$ can be calculated using (A.2):

The following is the case where $\tau$ is complete and the loading effect is sustained owing to external forces and drill bit inertia. Applying the conditions of $\tau<t \leq t_{m}, \sigma_{i}=0$, $F=k u, u_{0}=u(\tau)$, and $\dot{u}_{0}=\dot{u}(\tau)$ to (A.1) give (A.3) where $u=0$. Last, we examine the unloading condition where $t_{m}<t, \sigma_{i}=0, F=\gamma k u-\gamma k u_{f}, u_{0}=u_{m}$, and $\dot{u}_{0}=0$. Equation (9) when unloading can be expressed as (A.4).

In this case, bit displacement $u=u_{f}$ is used to indicate the rock's fracture displacement. Furthermore, considering the bit's damping ratio when unloading, its behavior follows

$$
\text { Damped mode }= \begin{cases}\zeta_{2}>1, & \alpha<\frac{1}{\gamma} \text { (no case), } \\ \zeta_{2}=1, & \alpha=\frac{1}{\gamma} \text { (no case), } \\ \zeta_{2}<1, & \alpha>\frac{1}{\gamma} \text { (under). }\end{cases}
$$

The second condition of critical damping is defined as $\alpha=1$. When unloading drill bits, $\gamma$ refers to the rock's property effect and is always less than 1 . Hence, when unloading, no other cases but the underdamped mode are allowed. In other words, when unloading in the critically damped mode owing to the rock properties, the bit is always underdamped.

The third condition is underdamping with $\zeta_{1}<1$ and $\alpha>1$. Here, $\tau$ is transmitted to the drill bit, meaning loading. Equation (7) can be expressed by (A.1). The initial conditions are $0<t \leq \tau, \sigma_{i}=0.5 \rho c v, F=k u, u_{0}=0$, and $\dot{u}_{0}=0$. Where, assuming the normal state, $u$ can be calculated as (A.2).

The following is the case where $\tau$ is complete, and the loading effect is sustained owing to external forces and drill bit inertia. Applying the conditions of $\tau<t \leq t_{m}, \sigma_{i}=0$, $F=k u, u_{0}=u(\tau)$, and $\dot{u}_{0}=\dot{u}(\tau)$ to (A.1) results in (A.3), where, $u=0$. Last, we examine the unloading condition where $t_{m}<t, \sigma_{i}=0, F=\gamma k u-\gamma k u_{f}, u_{0}=u_{m}$, and $\dot{u}_{0}=0$. Equation (9) when unloading can be expressed as (A.4).

In this case, the drill bit displacement $u=u_{f}$ is used to indicate the rock's fracture displacement. Furthermore, considering the damping ratio in the drill bit's unloading, the bit's behavior can be expressed as

$$
\text { Damped mode }= \begin{cases}\zeta_{2}>1, & \alpha<\frac{1}{\gamma} \text { (no case), } \\ \zeta_{2}=1, & \alpha=\frac{1}{\gamma} \text { (no case), } \\ \zeta_{2}<1, & \alpha>\frac{1}{\gamma} \text { (under). }\end{cases}
$$

The third condition is the critically damped mode defined as $\alpha>1$. During drill bit unloading, $\gamma$ refers to the rock property effect and is always less than 1. Hence, when unloading, no other cases but underdamping are allowed.

Next, the underdamped condition is where $\zeta_{1}<1$ and $\alpha>1$, and $\tau$ is slower than $t_{m}$ (the time at which the rock's maximum fracture displacement is reached), that is, $t_{m}<\tau$. In this case, $\tau$ is transmitted to the drill bit, meaning loading. Equation (7) can be expressed by (A.1). The initial conditions are $0<t \leq t_{m}, \sigma_{i}=0.5 \rho c v, F=k u, u_{0}=0$, and $\dot{u}_{0}=0$. Here, assuming the normal state, $u$ can be calculated as (A.2).

The following case is where conversion is made to unloading after the drill bit reaches its maximum displacement, but the incident stress wave is sustained. Applying the conditions of $t_{m}<t \leq \tau, \sigma_{i}=0.5 \rho c v$, $F=\gamma k u-\gamma k u_{f}, u_{0}=u_{m}$, and $\dot{u}_{0}=0$ to (9) results in

$$
\ddot{u}+\left(\frac{\rho c A}{m_{b}}\right) \dot{u}+\gamma\left(\frac{k}{m_{b}}\right) u=\left(\frac{\rho c A}{m_{b}}\right) v+\gamma\left(\frac{k}{m_{b}}\right) u_{f},
$$

where the definition of $u=1 / \gamma(\rho c A / k) v+u_{f}$ is possible.

Last, this unloading condition is where the incident stress wave is resolved. When unloading the drill bit, using the conditions $t<\tau, \sigma_{i}=0, F=\gamma k u-\gamma k u_{f}, u_{0}=u(\tau)$, and $\dot{u}_{0}=\dot{u}(\tau)$ allows (9) to be expressed as (A.4).

In this case, drill bit displacement $u=u_{f}$ is used to indicate the rock's fracture displacement. The analysis of drill bit dynamics showed that when loading transitions to unloading in the underdamped condition, the dynamic properties of the bit exist only in the underdamping mode.

\section{Conflicts of Interest}

The authors declare that there are no conflicts of interest.

\section{References}

[1] C. H. Song, K. B. Kwon, J. Y. Park et al., "Optimum design of the internal flushing channel of a drill bit using RSM and CFD simulation," International Journal of Precision Engineering and Manufacturing, vol. 15, no. 6, pp. 1041-1050, 2014. 
[2] X. Li, G. Rupert, D. A. Summers, P. Santi, and D. Liu, "Analysis of impact hammer rebound to estimate rock drillability," Rock Mechanics and Rock Engineering, vol. 33, no. 1, pp. 1-13, 2000.

[3] W. A. Hustrulid and C. Fairhurst, "A theoretical and experimental study of the percussive drilling of rock, part I: theory of percussive drilling," International Journal of Rock Mechanics and Mining Sciences \& Geomechanics Abstracts, vol. 8, no. 4, pp. 311-333, 1971.

[4] W. A. Hustrulid and C. Fairhurst, "A theoretical and experimental study of the percussive drilling of rock, part II: force-penetration and specific energy determination," International Journal of Rock Mechanics and Mining Sciences \& Geomechanics Abstracts, vol. 8, no. 4, pp. 335-356, 1971.

[5] W. A. Hustrulid and C. Fairhurst, "A theoretical and experimental study of the percussive drilling of rock, part III: experimental verification of the mathematical theory," International Journal of Rock Mechanics and Mining Sciences \& Geomechanics Abstracts, vol. 9, no. 3, pp. 417-418, 1972.

[6] W. A. Hustrulid and C. Fairhurst, "A theoretical and experimental study of the percussive drilling of rock, part IV: application of the model to actual percussive drilling," International Journal of Rock Mechanics and Mining Sciences, vol. 9, no. 3, pp. 431-442, 1972.

[7] L. E. Chiang and D. A. Elias, "A 3D FEM methodology for simulating the impact in rock-drilling hammers," International Journal of Rock Mechanics and Mining Sciences, vol. 45, no. 5, pp. 701-711, 2008.

[8] C. H. Song, K. B. Kwon, M. G. Cho, J. Y. Oh, D. Y. Shin, and J. W. Cho, "Development of lab-scale rock drill apparatus for testing performance of a drill bit," International Journal of Precision Engineering and Manufacturing, vol. 16, no. 7, pp. 1402-1414, 2015.

[9] K. B. Kwon, C. H. Song, J. Y. Park, J. Y. Oh, J. W. Lee, and J. W. Cho, "Evaluation of drilling efficiency by percussion testing of a drill bit with new button arrangement," International Journal of Precision Engineering and Manufacturing, vol. 15, no. 6, pp. 1063-1068, 2014.

[10] B. Lundberg and M. Okrouhlik, "Influence of 3D effects on the efficiency of percussive rock drilling," International Journal of impact Engineering, vol. 25, no. 4, pp. 345-360, 2001.

[11] B. Lundberg and P. Collet, "Optimal wave shape with respect to efficiency in percussive drilling with detachable drill bit," International Journal of impact Engineering, vol. 86, pp. 179187, 2015.

[12] X. B. Li, G. Rupert, and D. A. Summers, "Energy transmission of down-hole hammer tool and its conditionality," Transactions of Nonferrous Metals Society of China, vol. 10, no. 1, pp. 109-111, 2000.

[13] J. W. Cho, S. Jeon, S. H. Yu, and S. H. Chang, "Optimum spacing of TBM disc cutters: a numerical simulation using the three-dimensional dynamic fracturing method," Tunnelling and Underground Space Technology, vol. 25, no. 3, pp. 230244, 2010.

[14] W. Changming, "An analytical study of percussive energy transfer in hydraulic rock drills," Mining Science and Technology, vol. 13, no. 1, pp. 57-68, 1991.

[15] Online Materials Information Resource: http://www.matweb. com/search/DataSheet.aspx?MatGUID=8bc5d558f4174e6082 ddf4966e382bd6\&ckck=1. 


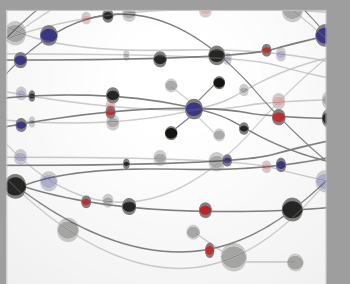

The Scientific World Journal
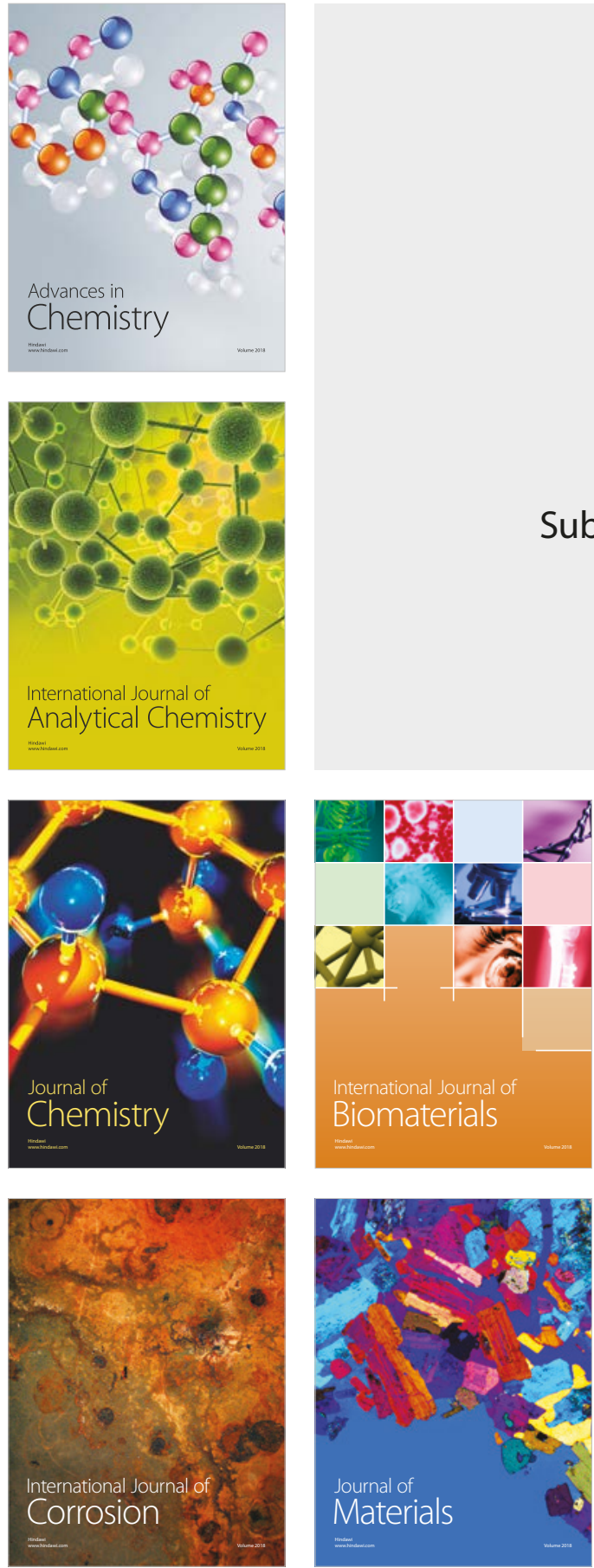

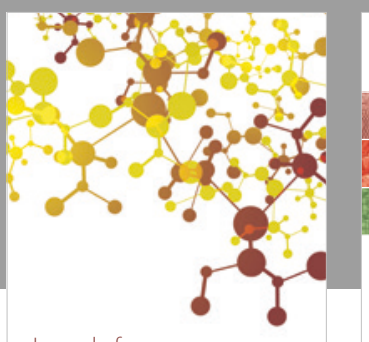

Journal of

Applied Chemistry
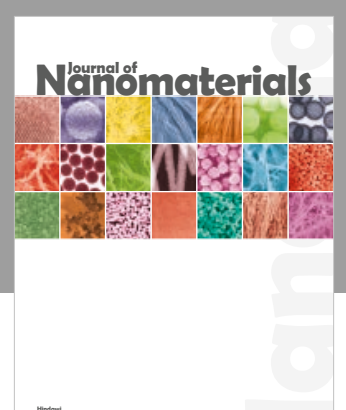

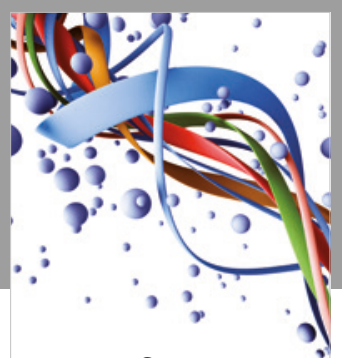

Scientifica

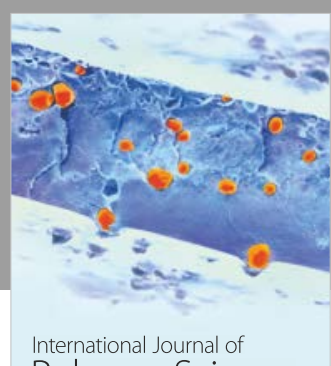

Polymer Science

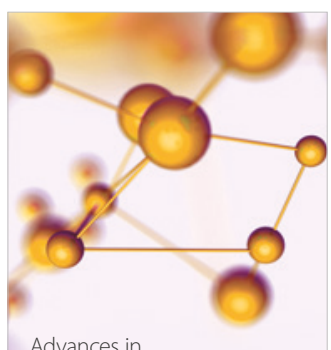

Physical Chemistry
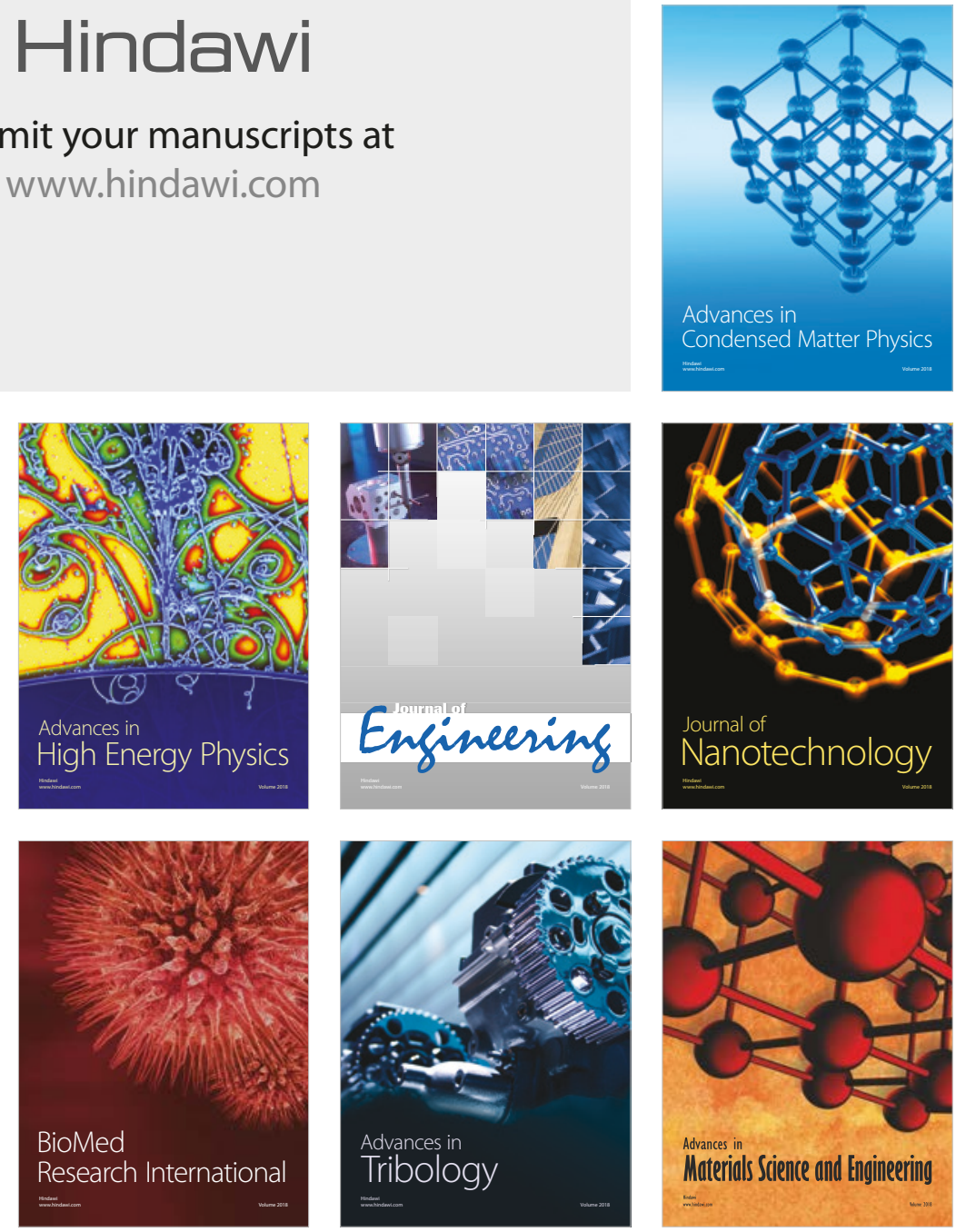